Acknowledgement: Yutaro Yamada

Disclosure of Interests: Yutaro Yamada Speakers bureau: Abbvie, Chugai, Mitsubishi Tanabe, Masahiro Tada Speakers bureau: Abbvie, Astellas Pharma, Bristol-Myers Squibb, Chugai Pharmaceutical, Eisai, Janssen Pharmaceutical, Mitsubishi Tanabe Pharma Corporation, Ono Pharmaceutical, Pfizer Japan, Takeda Pharmaceutical, Koji Mandai: None declared, Noriaki Hidaka: None declared, Kentaro Inui Speakers bureau: Takeda Pharmaceutical, Pfizer Japan, Daiichi-Sankyo Co.Ltd., Abbvie, Mitsubishi Tanabe Pharma Corporation, Janssen Pharmaceutical, Chugai Pharmaceutical, Ono Pharmaceutical, Eisai Co.Ltd., Eli-Lilly, Nippon Kayaku Co., Ltd., Maruho Co.,Ltd, Kaken Pharmaceutical Co.,Ltd., Hiroaki Nakamura: None declared

DOI: 10.1136/annrheumdis-2019-eular.1430

\section{FRI0073 $\quad$ SWELLING OR TENDERNESS, WHICH ONE CORRESPONDS BETTER WITH ULTRASOUND- DETECTED SYNOVITIS?}

Xiaoying Sun, Xuerong Deng, Wenhui Xie, Yu Wang, Zhuoli Zhang. Peking University First Hospital, Beijing, China

Background: Ultrasound (US) is a sensitive method for detecting joint inflammation in patients with rheumatoid arthritis (RA). The relationship between tender or swollen joints and ultrasound-detected synovitis has not been well explored in patients with RA

Objectives: The purpose of the present study was to compare the correlation between ultrasound-detected synovitis and joint tenderness or swelling at the wrists and hands in RA patients.

Methods: Twenty-two joints, including bilateral wrists, proximal interphalangeal (PIP) and metacarpophalangeal (MCP) joints, were respectively evaluated by physical examination (PE) and ultrasound scan in $258 \mathrm{RA}$ patients. All patients had at least 1 tender or swollen joint out of 22 joints. Synovitis was detected by using semi-quantitative scoring systems (0-3) for grey scale(GS) and power Doppler(PD). Positive synovitis was defined as $G S \geq 2$ and/or $P D \geq 1$. All correlations among US variables and clinical variables were assessed using Spearman's rank correlation test. Cohen's kappa ( $\kappa)$ between clinical and sonographic findings was calculated.

Results: Their median age was 51.2 years, median disease duration was 57 months, with $83.33 \%$ females. The mean (SD) Disease Activity Score based on 28 joints (DAS28)-ESR and DS28-CRP were $4.47 \pm 1.62$ and $3.99 \pm 1.51$, respectively. In a total of 5676 joints assessed, 968 swollen joints (17.05\%) and 1296 tender joints (22.83\%) were found, while on ultrasonography GS synovial hyperplasia was present in 801 (14.11\%) joints, positive PD in $476(8.38 \%)$ joints. There were more tender joints without swelling $(n=574)$ than those swollen joints without tenderness $(n=246)$. In all joints, higher $\kappa$ coefficient was observed in joint swelling $(\kappa=0.367, p<0.01)$ with ultrasound-detected synovitis compared with tenderness $(\kappa=0.281, p<0.01)$.Similarly, swollen joint counts of 22 joints showed higher Spearman's correlation coefficient with either total GS scores $(r=0.499, p<0.01)$ or total $P D$ scores of 22 joints $(r=0.430, p<0.01)$ than tender joint counts of 22 joints $(r=0.354 / 0.308, p<0.01)$. GS and PD synovitis were more frequently detected in swollen joints without tenderness compared with tender joints without swelling. This discrepancy tend to be more significant in MCP2, MCP3, and MCP4 joints $(p<0.05)$.

Conclusion: In RA patients with at least 1 tender or swollen joint of wrists and hands, a higher frequency of joint tenderness was observed than swelling. However, swelling had better agreement with ultrasound detected synovitis compared with tenderness. Joint swelling is more associated with ultrasound-detected synovitis than tenderness. Without swelling, joint tenderness tends to be less associated with ultrasound-detected synovitis.

\section{REFERENCE:}

[1] Tan YK, Moorakonda RB, Allen JC Jr, Chew LC, Thumboo J. Back to the basics: Understanding joint swelling and tenderness at the wrist in rheumatoid arthritis through the use of ultrasonography. Int J Rheum Dis. 2018 Oct 21.
Acknowledgement: The authors thank all the colleagues in our department for kind cooperation in this project and the patients for participating in this study.

Disclosure of Interests: None declared

DOI: 10.1136/annrheumdis-2019-eular.3601

\section{Rheumatoid arthritis - biological DMARDs}

\section{FRI0074 UPDATE FOR THE CLINICAL PRACTICE: INTEGRATED, EVIDENCE-BASED APPROACH FOR THE MANAGEMENT OF RHEUMATOID ARTHRITIS}

Mohammed Hassan Abu-Zaid ${ }^{1}$, Yasser El Miedany ${ }^{2}$, Mohammed A Mortada ${ }^{3}$, Waleed Hassan ${ }^{4}$, Mervat Eissa ${ }^{5}$, Yossra Atef ${ }^{6}$, Samah Ismail Nasef ${ }^{7}$, Hala Lotfy ${ }^{8}$, Yomna Farag ${ }^{8}$, Ghada El Deriny ${ }^{9}$, Nadia El Aroussy ${ }^{10}$, Maha El Gaafary ${ }^{11}$ Dalia Mekkawy ${ }^{10} .{ }^{1}$ Rheumatology and Rehabilitation, School of Medicine Tanta University, Tanta, Egypt, ${ }^{2}$ Rheumatology, Darent Valley Hospital, Dartford, UK, Dartford, United Kingdom; ${ }^{3}$ Rheumatology and Rehabilitation, School of Medicine Zagazig University, Zagazig, Egypt, ${ }^{4}$ Rheumatology and Rehabilitation, School of Medicine Banha University, Banha, Egypt, ${ }^{5}$ Rheumatology and Rehabilitation, School of Medicine Cairo University, Cairo, Egypt, ${ }^{6}$ Rheumatology and Rehabilitation, School of Medicine Assiut University, Assiut, Egypt, ${ }^{7}$ Rheumatology and Rehabilitation, School of Medicine Suez Canal University, Ismaillia, Egypt,

${ }^{8}$ Pediatrics, School of Medicine Cairo University, Cairo, Egypt, ${ }^{9}$ Pediatrics, School of Medicine Alexandria University, Alexandria, Egypt ${ }^{10}$ Rheumatology and Rehabilitation, School of Medicine Ain Shams University, Cairo, Egypt,

${ }^{11}$ Community and Public Health, School of Medicine Ain Shams University, Cairo, Egypt

Background: The management of rheumatoid arthritis has evolved in the last few years with greater emphasis on a treat-to-target strategy rather than specific drug regimens.Technologies such as ultrasound have been increasingly used for diagnosis and monitoring of synovitis where it is unclear from clinical examination. These aspects of management were investigated by the Guideline Committee, and recommendations have been updated using new evidence [1,2].

Objectives: To develop an updated evidence-based, interdisciplinary recommendations for the management of rheumatoid arthritis (RA).

Methods: The task force comprised rheumatologists, epidemiologists, health economists, and patients. A systematic literature analysis was conducted using the Cochrane Database of Systematic Reviews and MEDLINE. Data regarding the drugs prices and product information were collected to evaluate drug cost and safety. Evidence and recommendations were graded, and an algorithm for treatment and final statements were discussed in a consensus meeting (Nominal Group Technique). Recommendations were developed using a Delphi method by a multidisciplinary panel including patients.

Results: Consensus was reached on recommendations, including a standardized treatment strategy according to the RA severity, activity and prog nostic factors in the individual patient. In this updated interdisciplinary guideline for RA the treat to target strategy was considered the cornerstone in these recommendations (figure 1). RA patients should reach clinical, ultrasonographic and functional remission. Treatment decisions should incorporate the disease activity status, structural damage progression (radiologic/sonographic), comorbidities, quality of life and patient motivation Physiatrist, psychologist and ocupational therapist should be included in interdiscibilnary treatment team for RA.

Conclusion: The treatment strategy included a total of twenty-one evidence based interdisciplinary recommendations for management of RA (figure 1), and is accompanied by a more in-depth discussion of key management principles. These recommendations provide a step-wise approach to treatment, to enable practitioners to develop and support the most effective method of achieving and maintaining remission in RA patients. These recommendations are not to remove the physician's autonomy, and physicians must select the most appropriate therapeutic option, taking into consideration the patient's preferences. 